\title{
The kinetic Shakhov-Enskog model for non-equilibrium flow of dense gases
}

\author{
Peng Wang ${ }^{1}$, Lei $\mathrm{Wu}^{1}$, Minh-Tuan $\mathrm{Ho}^{1}$, Jun $\mathrm{Li}^{2}$, Zhi-Hui $\mathrm{Li}^{3}$ and \\ Yonghao Zhang ${ }^{1} \dagger$ \\ ${ }^{1}$ James Weir Fluids Laboratory, Department of Mechanical and Aerospace Engineering, \\ University of Strathclyde, Glasgow G1 1XJ, UK \\ ${ }^{2}$ Center for Integrative Petroleum Research, College of Petroleum Engineering and \\ Geosciences, King Fahd University of Petroleum and Minerals, Saudi Arabia \\ ${ }^{3}$ Hypervelocity Aerodynamics Institute, China Aerodynamics Research and Development \\ Center, Mianyang 621000, China
}

(Received xx; revised xx; accepted xx)

When the average intermolecular distance is comparable to the size of gas molecules, the Boltzmann equation, based on the dilute gas assumption, becomes invalid. The Enskog equation was developed to account for this finite size effect that makes the collision non-local and increases the collision frequency. However, it is time-consuming to solve the Enskog equation due to its complicated structure of collision operator and high dimensionality. In this work, on the basis of the Shakhov model, a gas kinetic model is proposed to simplify the Enskog equation for non-ideal monatomic dense gases. The accuracy of the proposed Shakhov-Enskog model is assessed by comparing its solutions of the normal shock wave structures with the results of the Enskog equation obtained by the fast spectral method. It is shown that the Shakhov-Enskog model is able to describe non-equilibrium flow of dense gases, when the maximum local mean free path of gas molecules is still greater than the size of molecular diameter. The accuracy and efficiency of the present model enable simulations of non-equilibrium flow of dense gases for practical applications.

Key words: Gas kinetic theory, Enskog equation, Boltzmann equation, rarefied gas dynamics

\section{Introduction}

In the past one and half centuries, the Boltzmann equation has achieved great success in describing the dynamics of rarefied ideal gases, that is, the gas is sufficiently dilute that only binary collisions need to be taken into account (Chapman \& Cowling 1970). However, if the gas is compressed to the level that the molecular dimensions are comparable to the average distance of the neighboring molecules, the effect of finite molecular size must be considered (Ferziger \& Kaper 1972). This is found in many applications including high pressure shock tubes (Petersen \& Hanson 2001), sonoluminescence (Brenner et al. 2002), gas extraction in unconventional reservoirs (Wu et al. 2016; Sander et al. 2017), and the interfacial dynamics of liquid-vapor at supercritical pressures (Dahms et al. 2013; Dahms \& Oefelein 2015).

$\dagger$ Email address for correspondence: yonghao.zhang@strath.ac.uk 
Enskog (1922) was the first to extend the Boltzmann equation to dense gases of rigid spherical molecules by considering the non-local collisions and the increased collision frequency due to the finite size of gas molecules (Chapman \& Cowling 1970; Ferziger \& Kaper 1972). Similar to the Boltzmann equation, the Enskog equation can be numerically solved using a probabilistic or deterministic method. The pioneering work of solving the Enskog equation was given by Frezzotti \& Sgarra (1993) using a Monte Carlo quadrature method, and its accuracy was confirmed by comparing with the Molecular Dynamics (MD) simulation of the shock wave structure in a dense hard-sphere gas. Subsequently, inspired by the direct simulation of Monte Carlo (DSMC) method for the Boltzmann equation, several probabilistic methods were developed by Alexander et al. (1995), Montanero \& Santos (1996), and Frezzotti (1997b) to solve the Enskog equation. Recently, a fast spectral method (FSM) method was proposed by Wu et al. (2015) to deterministically solve the generalized Enskog equation, which has spectral accuracy in computing the collision operator at reduced computational cost, and has been successfully applied to study the non-equilibrium dynamics of dense gas under tight confinement (Wu et al. 2016). More recently, Sadr \& Gorji (2017, 2019) also developed the Fokker-Planck approximation of the Enskog equation for non-equilibrium dense gases, which was validated by comparing with the results of the Enskog model solved by the Monte Carlo method (Montanero \& Santos 1996).

Although these methods for direct solution of the Enskog equation could provide reliable results, the computational costs are still formidable for practical applications. Therefore, it is beneficial to simplify the Enskog collision operator to achieve efficient computations with reasonable accuracy. The first attempt, although not designed for numerical simulations, was made in the derivation of transport coefficients such as viscosity and thermal conductivity, where the Enskog collision operator was expanded into the Taylor series of molecular diameter. The zeroth-order term gave the Boltzmann collision operator, and in the first-order terms, the velocity distribution function (VDF) was approximated by the local equilibrium distribution function, while the other high-order terms were neglected (Chapman \& Cowling 1970). In developing the lattice Boltzmann model (LBM) for hydrodynamic flows of both liquid and gas, Luo (1998, 2000) borrowed and simplified the Enskog equation by replacing the Boltzmann collision operator with a Bhatnagar-Gross-Krook (BGK) collision operator (Bhatnagar et al. 1954), while maintaining the first-order terms relevant to the density spatial derivative. Later, by including the long-range attractive force, the BGK-Enskog model was used to develop LBM multiphase model by He \& Doolen (2002). However, the above LBM works only considered hydrodynamic flows. As a consequence of the inherent coupling between the discretized molecular velocity and spatial grid in the standard LBM, the limited number of discrete velocities in LBM results in poor numerical stability and its simulation is limited to low-speed flow (Guo \& Shu 2013). Hence, these LBM models are not appropriate for simulating compressible flows. Subsequently, Guo et al. (2005, 2006) proposed a simplified kinetic model for strongly inhomogeneous confined flow by considering the attractive force among molecules and the wall potential, from which the generalized hydrodynamic equations for fluid flows from nanoscale to macroscale were derived based on the Chapman-Enskog expansion.However, Guo et al. (2005, 2006) only studied equilibrium flow properties with no-slip boundary condition, so non-equilibrium effects were not properly considered. Here, we aim to propose an accurate and efficient model equation to describe rarefied flow of dense gases that underpin many technological developments.

In this work, a simplified model of the Enskog equation is to be developed on the basis of the Shakhov model for dense gases of the monatomic rigid molecules, which is 
presented in $\S 2$. In order to test its accuracy, the structure of normal shock wave in a dense gas is calculated by the proposed model, and the results are compared to the solutions of Enskog equation using the fast spectral method, which are presented in $\S 3$.

\section{The Shakhov-Enskog equation}

\subsection{The Enskog equation}

To describe non-equilibrium (rarefied) flow of dilute gases, the Boltzmann equation assumes that the collisions between molecules occur instantaneously and only twobody interaction exists between molecules. In the time between two collisions, the molecule is only affected by external force, and its motion is independent of other molecules. The collisions between molecules are statistically independent. When the gases become dense, the space occupied by molecules needs to be considered, and molecular transport cannot be completely described by the free motion between two consecutive collisions. Enskog (1922) studied the transport of dense gases comprising of rigid, elastic, and spherical molecules, and developed the Enskog-Boltzmann equation with the following two arguments. First, when two molecules collide, their positions are not at the same point, but one molecular diameter away. As binary collisions are assumed in the Enskog equation, it is only applicable for moderately dense gases. Second, the volume occupied by the gas molecules reduces the space of free movement of each molecule, and hence the number of collisions between molecules increases. As a further approximation, the collision operator of the Boltzmann equation can be modified and extended to the Enskog equation for hard-sphere molecules as (Chapman \& Cowling 1970):

$$
\frac{\partial f}{\partial t}+\boldsymbol{\xi} \cdot \nabla_{x} f+\boldsymbol{a} \cdot \nabla_{\xi} f=\Omega,
$$

where $f(\boldsymbol{x}, \boldsymbol{\xi}, t)$ is the VDF of the molecular velocity $\boldsymbol{\xi}$ at position $\boldsymbol{x}$ and time $t, m \boldsymbol{a}$ is the external body force ( $m$ is the molecular mass), $\nabla_{x}$ and $\nabla_{\xi}$ are the gradient operator with respect to the physical space and molecular velocity, respectively. On the right-hand side of (2.1), $\Omega$ is the Enskog collision operator with the form of

$$
\Omega=\sigma^{2} \int\left\{\chi\left(\boldsymbol{x}+\frac{\sigma}{2} \boldsymbol{k}\right) f^{\prime}(\boldsymbol{x}) f_{1}^{\prime}(\boldsymbol{x}+\sigma \boldsymbol{k})-\chi\left(\boldsymbol{x}-\frac{\sigma}{2} \boldsymbol{k}\right) f(\boldsymbol{x}) f_{1}(\boldsymbol{x}-\sigma \boldsymbol{k})\right\} \boldsymbol{g} \cdot \boldsymbol{k} d \boldsymbol{k} d \boldsymbol{\xi}_{\mathbf{1}} .
$$

Here $\sigma$ is the effective molecular diameter, $\boldsymbol{g}=\boldsymbol{\xi}_{1}-\boldsymbol{\xi}$ is the relative velocity of two colliding molecules, and $\boldsymbol{k}$ is the unit vector that specifies their relative position at the time of impact. In addition, $f^{\prime}(\boldsymbol{x}) \equiv f\left(\boldsymbol{x}, \boldsymbol{\xi}^{\prime}, t\right), f_{1}^{\prime}(\boldsymbol{x}) \equiv f\left(\boldsymbol{x}, \boldsymbol{\xi}_{1}^{\prime}, t\right), f_{1}(\boldsymbol{x}) \equiv f\left(\boldsymbol{x}, \boldsymbol{\xi}_{1}, t\right)$, and $f(\boldsymbol{x}) \equiv f(\boldsymbol{x}, \boldsymbol{\xi}, t)$, where the post-collision velocities $\boldsymbol{\xi}^{\prime}$ and $\boldsymbol{\xi}_{1}^{\prime}$ are related to the precollision molecular velocities $\boldsymbol{\xi}$ and $\boldsymbol{\xi}_{1}$ through $\boldsymbol{\xi}^{\prime}=\boldsymbol{\xi}+\boldsymbol{k}(\boldsymbol{g} \cdot \boldsymbol{k})$ and $\boldsymbol{\xi}_{1}^{\prime}=\boldsymbol{\xi}_{1}-\boldsymbol{k}(\boldsymbol{g} \cdot \boldsymbol{k})$. In comparison with the Boltzmann equation, a radial distribution function (RDF) $\chi$ is introduced in the Enskog collision operator (2.2) to include the effect of physical dimensions of the molecules on the intermolecular collision frequency. The RDF depends on the local density $\rho(\boldsymbol{x}, t)$ and can be approximated by (Carnahan \& Starling 1969; Chapman \& Cowling 1970)

or

$$
\chi(\rho)=\frac{1-0.125 b \rho}{(1-0.25 b \rho)^{3}},
$$

$$
\chi(\rho)=\frac{1-(11 / 8) b \rho}{1-2 b \rho} .
$$


In (2.3) and (2.4), $b=2 \pi \sigma^{3} / 3 m$, which relates to the reduced density $\eta$ as $\eta=b \rho / 4$. Note that (2.4) is only used in this work for consistent comparison with the reported solutions of the Enskog equation obtained by the Monte Carlo method (Frezzotti \& Sgarra 1993). In comparison with (2.4), the RDF defined by (2.3) agrees extremely well with the MD data given by Alder \& Wainwright (1960) up to the phase transition. Therefore, (2.3) will be applied for the rest of simulations. When $\sigma$ approaches zero, $\chi$ is unity, and the Enskog collision operator (2.2) reduces to that of the Boltzmann equation. It is apparent that direct numerical solution of the Enskog equation (2.1) is even more time consuming than the Boltzmann equation, restricting its practical applications. Therefore, it is important to simplify the Enskog equation to improve computational efficiency while still satisfying accuracy requirement for dense gases.

\subsection{The Shakhov-Enskog model}

Here, a kinetic model of the Enskog equation is derived for non-equilibrium flow of dense gases on the basis of the Shakhov model, which is able to represent a correct Prandtl number $(\mathrm{Pr})$ by expanding the Maxwellian velocity distribution function in the BGK model (Bhatnagar et al. 1954). Assuming the gas flow is sufficiently smooth, the right hand side of $(2.1)$ can be approximated by expanding $\chi\left(\boldsymbol{x} \pm \frac{\sigma}{2} \boldsymbol{k}\right), f_{1}(\boldsymbol{x} \pm \sigma \boldsymbol{k})$ and $f_{1}^{\prime}(\boldsymbol{x} \pm \sigma \boldsymbol{k})$ in a Taylor series around $\boldsymbol{x}$, retaining only the first-order derivatives; the Enskog equation without the external body force term can therefore be written as (Chapman \& Cowling 1970; Ferziger \& Kaper 1972; Kremer 2010)

$$
\frac{\partial f}{\partial t}+\boldsymbol{\xi} \cdot \nabla f=J^{0}(f f)+J_{a}^{1}(f f)+J_{b}^{1}(f f),
$$

where

$$
\begin{aligned}
& J^{0}(f f)=\chi \int\left[f^{\prime} f_{1}^{\prime}-f f_{1}\right] \sigma^{2} \boldsymbol{g} \cdot \boldsymbol{k} d \boldsymbol{k} d \boldsymbol{\xi}_{1}, \\
& J_{a}^{1}(f f)=\frac{\sigma}{2} \int \boldsymbol{k} \cdot \nabla \chi\left[f^{\prime} f_{1}^{\prime}+f f_{1}\right] \sigma^{2} \boldsymbol{g} \cdot \boldsymbol{k} d \boldsymbol{k} d \boldsymbol{\xi}_{1}, \\
& J_{b}^{1}(f f)=\sigma \chi \int \boldsymbol{k} \cdot\left[f^{\prime} \boldsymbol{\nabla} f_{1}^{\prime}+f \nabla f_{1}\right] \sigma^{2} \boldsymbol{g} \cdot \boldsymbol{k} d \boldsymbol{k} d \boldsymbol{\xi}_{1} .
\end{aligned}
$$

In (2.6), the quantities of $f, f_{1}, f^{\prime}, f_{1}^{\prime}$ and $\chi$ are evaluated at the point $\boldsymbol{x}$. Note that, $J^{0}(f f)$ is a modification of the usual collision operator of the Boltzmann equation with a factor of $\chi$ in its numerator, which can therefore be simplified as that for the Boltzmann collision operator.

With the aim of simplifying the structure of $J^{0}(f f)$ but maintaining its basic properties, simpler expressions for the collision term were introduced in the literature, which are known as the kinetic models. In principle, a kinetic model must fulfill the same properties of the original collision operator, i.e., (i) for all summation invariants $\boldsymbol{\psi}=\left(1, \boldsymbol{\xi}, \frac{1}{2} \xi^{2}\right)^{T}$, the collision operator must satisfy the conservation laws, i.e.

$$
\int \boldsymbol{\psi} J^{0}(f, f) d \boldsymbol{\xi}=0
$$

(ii) in equilibrium, the VDF should be a Maxwellian; (iii) production of entropy is always positive, satisfying the $\mathcal{H}$-theorem, i.e.

$$
\int \ln f J^{0}(f, f) d \boldsymbol{\xi} \leqslant 0
$$

The same as a dilute gas, the gas molecules in a dense gas will also tend to approach 
equilibrium through collisions. Thereby, the kinetic model for dilute gases may be borrowed to simplify the Enskog equation. The Shakhov model is among the most popular kinetic models (Titarev 2018; Zhu et al. 2019; Venugopal et al. 2019), although its $\mathcal{H}$-theorem (2.8) has not been proved (Shakhov 1968). Therefore, the Shakhov model relaxation operator is readily adopted to simplify $J^{0}(f f)$ (Shakhov 1968), which reads

$$
J^{0}(f f)=-\frac{1}{\tilde{\tau}}\left(f-f^{S}\right)
$$

with $\tilde{\tau}$ being the relaxation time of dense gases, and $f^{S}$ being the reference VDF expressed by the Maxwellian distribution function $f^{e q}$ and a heat flux correction term:

$$
\left.f^{S}=f^{e q}\left[1+(1-\operatorname{Pr}) \frac{\boldsymbol{c} \cdot \boldsymbol{q}}{5 p_{0} R T}\left(\frac{c^{2}}{R T}-5\right)\right)\right] .
$$

In (2.10), the Prandtl number is set to be $2 / 3$ for the monatomic gases, $\boldsymbol{c}=\boldsymbol{\xi}-\boldsymbol{u}$ is the peculiar molecule velocity, and $\boldsymbol{q}=\frac{1}{2} \int \boldsymbol{c} c^{2} f \mathrm{~d} \boldsymbol{\xi}$ is the heat flux due to the translational motion of gas molecules; $p_{0}=\rho R T$ is the equation of state for ideal gas with $R$ being the specific gas constant; $\rho, \boldsymbol{u}$, and $T$ are the flow density, macroscopic velocity, and temperature, respectively. The Maxwellian distribution function $f^{e q}$ is given by

$$
f^{e q}=\frac{\rho}{(2 \pi R T)^{3 / 2}} \exp \left(-\frac{c^{2}}{2 R T}\right) .
$$

While in $J_{a}^{1}(f f)$ and $J_{b}^{1}(f f)$, the VDFs can be approximated by the corresponding Maxwell distribution functions. Using $f^{e q^{\prime}} f_{1}^{e q^{\prime}}=f^{e q} f_{1}^{e q}$, one can obtain the formulation of (Chapman \& Cowling 1970)

$$
\begin{aligned}
\Omega_{E} \equiv J_{a}^{1}\left(f^{e q} f^{e q}\right)+J_{b}^{1}\left(f^{e q} f^{e q}\right)= & -b \rho \chi f^{e q}\left\{\boldsymbol{c} \cdot\left[\nabla \ln \left(\rho^{2} \chi T\right)+\frac{3}{5}\left(\zeta^{2}-\frac{5}{2}\right) \nabla \ln T\right]\right. \\
& \left.+\frac{2}{5}\left[2 \zeta \zeta: \nabla \boldsymbol{u}+\left(\zeta^{2}-\frac{5}{2}\right) \boldsymbol{\nabla} \cdot \boldsymbol{u}\right]\right\}
\end{aligned}
$$

where $\boldsymbol{\zeta}=\boldsymbol{c} / \sqrt{2 R T}$. In order to obtain (2.12), the integrations have been made with respect to $\boldsymbol{k}$ and $\boldsymbol{\xi}_{1}$. It should be noted that, for dense gases, collisions between gas molecules also contribute to transfer of momentum and energy.

Finally, the Enskog equation based on the Shakhov collision model with the absence of the external force can be derived as

$$
\frac{\partial f}{\partial t}+\boldsymbol{\xi} \cdot \nabla f=-\frac{1}{\tilde{\tau}}\left[f-f^{S}\right]+\Omega_{E}
$$

Once the VDF is obtained, the conservative variables $\boldsymbol{W} \equiv(\rho, \rho \boldsymbol{u}, \rho \epsilon)^{T}$ are calculated as

$$
\boldsymbol{W}=\int \boldsymbol{\psi} f \mathrm{~d} \boldsymbol{\xi}
$$

The gas temperature is related to the total gas energy as $\rho \epsilon=\frac{1}{2} \rho u^{2}+e_{k}$, with $e_{k}=$ $3 \rho R T / 2$ being the kinetic energy density.

The determination of relaxation time parameter $\tilde{\tau}$ in (2.13) is critically important for the kinetic model, as it affects the momentum and heat transfer processes during the intermolecular collisions. Through the Chapman-Enskog expansion of (2.13), we can 
obtain the conservative equations for mass, momentum, and energy as

$$
\begin{aligned}
\partial_{t} \rho+\boldsymbol{\nabla} \cdot(\rho \boldsymbol{u}) & =0, \\
\partial_{t}(\rho \boldsymbol{u})+\boldsymbol{\nabla} \cdot(\rho \boldsymbol{u u}) & =-\nabla p+\nabla \cdot\left(2 \tilde{\tau} p_{0} \boldsymbol{S}\right), \\
\partial_{t}\left(e_{k}\right)+\boldsymbol{\nabla} \cdot\left(\boldsymbol{u} e_{k}\right) & =-p \boldsymbol{\nabla} \cdot \boldsymbol{u}+\boldsymbol{\nabla} \cdot(\lambda \boldsymbol{\nabla} T)+2 \tilde{\tau} p_{0} \boldsymbol{S}: \boldsymbol{\nabla} \boldsymbol{u},
\end{aligned}
$$

where $p=p_{0}(1+b \rho \chi)$ is hydrostatic pressure for non-ideal gas, $\lambda$ is the coefficient of thermal conductivity, and $\boldsymbol{S}$ is the rate of shear tensor

$$
S_{\alpha \beta}=\frac{1}{2}\left(\frac{\partial U_{\beta}}{\partial x_{\alpha}}+\frac{\partial U_{\alpha}}{\partial x_{\beta}}\right)-\frac{1}{3} \nabla \cdot \boldsymbol{u} \delta_{\alpha \beta},
$$

with $U_{\alpha, \beta}$ being the components of the flow velocity, where $\alpha, \beta \in(x, y, z)$. As a consequence, it is readily to see that the coefficient of shear viscosity $\mu=\tilde{\tau} p_{0}$; hence the relaxation time can be defined as

$$
\tilde{\tau}=\frac{\mu}{p_{0}}
$$

where the viscosity coefficient is taken as $\mu=\mu_{0} b \rho\left[(b \rho \chi)^{-1}+0.8+0.7614 b \rho \chi\right]$ (Chapman \& Cowling 1970). Here, the coefficient of viscosity $\mu$ is obtained through the firstorder Chapman-Enskog expansion of the Enskog equation (Chapman \& Cowling 1970), which accounts for both the kinetic and potential contributions; therefore, the relaxation time of (2.17) may accurately describe the collisional transfer due to the non-local molecular collisions. Here $\mu_{0}(T)=\mu_{r e f}\left(T / T_{1}\right)^{0.5}$ is the viscosity coefficient for hardsphere molecules, with $\mu_{\text {ref }}$ being the reference viscosity coefficient for the dilute gases at the temperature $T_{1}$.

Intuitively, since the $J_{0}$ is just replaced by the kinetic model for dilute gas, the relaxation time may be inherited directly from the kinetic model, i.e.

$$
\tilde{\tau}=\frac{\mu_{1}}{p_{0}}
$$

with $\mu_{1}=\mu_{0} / \chi$. Note that the relaxation time of $(2.18)$ is exactly the same as the one given by Luo $(1998,2000)$ for the simplified Enskog model based on the BGK model (here denoted as the BGK-Enskog model) for isothermal low-speed flows. Later, for the BGK-Enskog model with the inclusion of the long range attractive force for multiphase flows, He \& Doolen (2002) proposed another formula of relaxation time as

$$
\tilde{\tau}=\frac{\mu_{2}}{p_{0}}
$$

with $\mu_{2}=(1+0.4 b \rho \chi) \mu_{0} / \chi$. Note that, the viscosity coefficient $\mu_{2}$ is extracted from the first-order kinetic contributions to the pressure tensor, which neglects the potential contributions to the transport of momentum and energy during molecular collisions. Consequently, the influence of the non-local collisions on the momentum and heat transfer may not be well described. We will also evaluate numerically for these different formulations of relaxation time.

Note that, when the Prandtl number in the Shakhov-Enskog model is set to be unity, $J_{0}(f f)$ will reduce to the collision operator of the BGK model, reading as

$$
J^{0}(f f)=-\frac{1}{\tilde{\tau}}\left(f-f^{e q}\right) .
$$

However, this BGK-Enskog model cannot properly describe the heat flux due to its inherited incorrect Prandtl number even for ideal gases. We will numerically examine 
this model as well by comparing with the Enskog equation and the Shakhov-Enskog model.

According to (2.13), the properties of Shakhov-Enskog model solely depend on the Shakhov collision term $J^{0}$ of $(2.9)$ and $\Omega_{E}$ of (2.12). While the Shakhov collision operator can capture non-equilibrium effects, (2.12) describes the collisional contribution to transfer of gas momentum and energy due to the dense gas effects. When the reduced density $\eta$ approaches zero (i.e., $\chi=1$ ), the proposed model reduces to the Shakhov model. While other kinetic models for dilute gases can be adopted for $J^{0}$ of (2.9), potential drawbacks of the Shakhov model are inherited including unproved $\mathcal{H}$-theorem of the Shakhov collision operator and possible negative value of VDF. Additionally, as a derived model from the Enskog equation, the proposed Shakhov-Enskog model is also valid only for moderately dense gases.

\section{Normal shock wave in dense gases}

The propagation of normal shock wave in a dense gas (Frezzotti 1997a; Frezzotti \& Sgarra 1993; de Haro \& Garzó 1995; Montanero et al. 1998, 1999) is ideal to test whether the Shakhov-Enskog model can capture the dense and highly non-equilibrium effects, since it is a spatially one-dimensional problem where the boundary effect, i.e. the influence from solid walls, is absent. Attention is given to the calculation of the fully formed shock profiles, which appear stationary to an observer moving with the shock front. In the normal shock wave, the upstream and downstream equilibrium states are connected by the following Rankine-Hugoniot relationships (Frezzotti \& Sgarra 1993):

$$
\begin{aligned}
\rho_{1} U_{1} & =\rho_{2} U_{2}, \\
\rho_{1}\left[U_{1}^{2}+R T_{1}\left(1+b \rho_{1} \chi_{1}\right)\right] & =\rho_{2}\left[U_{2}^{2}+R T_{2}\left(1+b \rho_{2} \chi_{2}\right)\right], \\
U_{1}^{2}+R T_{1}\left(5+2 b \rho_{1} \chi_{1}\right) & =U_{2}^{2}+R T_{2}\left(5+2 b \rho_{2} \chi_{2}\right),
\end{aligned}
$$

where the flow variables with subscriptions ' 1 ' and ' 2 ' represent the flow field at upstream and downstream of the shock, respectively.

\subsection{Algorithm and computational parameters setup}

In this study, the Enskog collision operator is calculated by the fast spectral method (Wu et al. 2015). The FSM for solving the Enskog equation for low-speed flows has already been well-studied by comparing with the analytical solutions, and the computational results of the Monte Carlo method and the molecular dynamics for several challenging cases (Wu et al. 2015, 2016), however, the capability of solving the Enskog equation by the FSM for the canonical normal shock wave in a dense gas remains unexplored. In the following, the results of the Enskog equation solved by the FSM are first validated by comparing with the solutions of the well-established MD and Monte Carlo method, thereafter the proposed Shakhov-Enskog model is evaluated by comparing with the FSM solution of the Enskog equation directly. Therefore, we can ensure consistent comparison between the model equation and the Enskog equation due to data scarcity for non-equilibrium flow of dense gases in the literature.

Note that the case of interest is one-dimensional problem (here the shock wave is assumed to be propagated along the $x$-direction), hence in order to reduce the computational cost, the variables along the other two directions can be reduced by making integration along these two axes; the details can be found in Appendix A. Many available discrete velocity methods (DVMs) can solve the proposed model properly. In this work, the iterative implicit DVM, which is simple and efficient, is employed to solve (2.13): given 


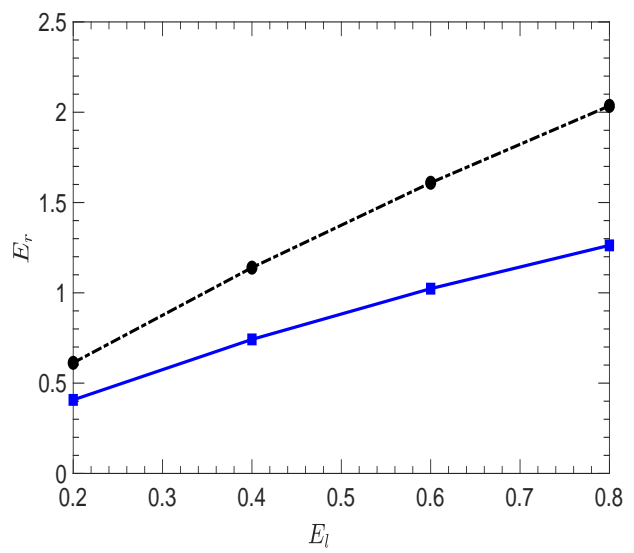

Figure 1: The downstream dimensionless parameter $E_{r}$ for $\mathrm{Ma}=2$ (solid line) and 4 (dash-dot line) against the upstream $E_{l}$.

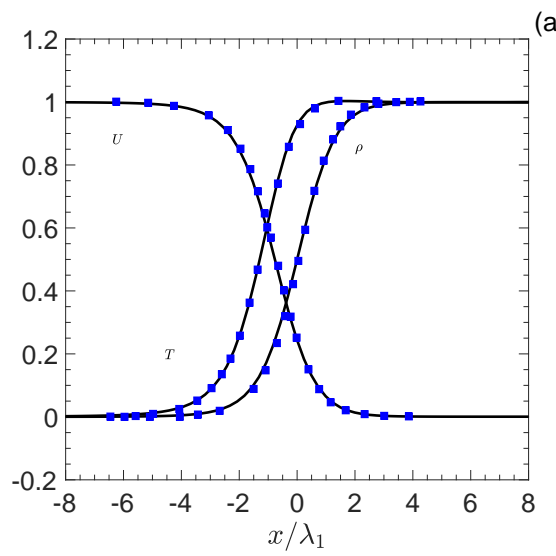

(a)

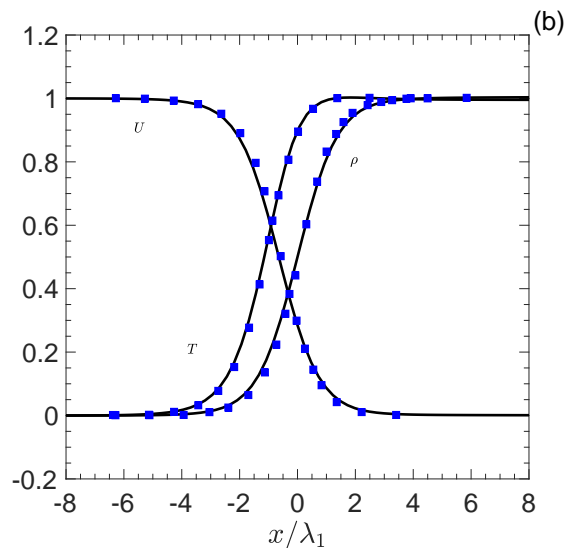

Figure 2: Comparison of the normalized $\rho, U$ and $T$, obtained from the Monte Carlo method by Frezzotti \& Sgarra (1993)(solid square symbols) and the fast spectral method (solid line) of solving the Enskog equation at $(a) \mathrm{Ma}=3$ and $E_{l}=0 ;(b) \mathrm{Ma}=4$ and $E_{l}=0.2$.

the value of $f$ at the $k$-th time step, its value at the next iteration step is calculated by the following equation (Yang \& Huang 1995; Wu et al. 2013)

$$
\xi_{x} \frac{\partial f^{k+1}}{\partial x}=-\frac{1}{\tilde{\tau}}\left[f^{k+1}-f^{S, k}\right]+\Omega_{E}^{k}
$$

where the spatial derivative is approximated by the second-order upwind scheme. The details of FSM solving the Enskog equation can be found in $\mathrm{Wu}$ et al. (2015). In order to accurately approximate the macroscopic flow moments on the velocity space at a few Mach numbers (Ma) and various degree of denseness, the computational parameters such as the spatial mesh size and the discretization of molecular velocity space should be appropriately chosen, until no appreciable changes in the solutions are observed.

The upstream $\rho_{1}$ and $T_{1}$ are chosen as the reference values of density and temperature, respectively. The flow velocity is normalized by the most probable velocity $v_{m}=\sqrt{2 R T_{1}}$. The upstream dimensionless parameter $E_{l}$, which was first introduced by Frezzotti \& 


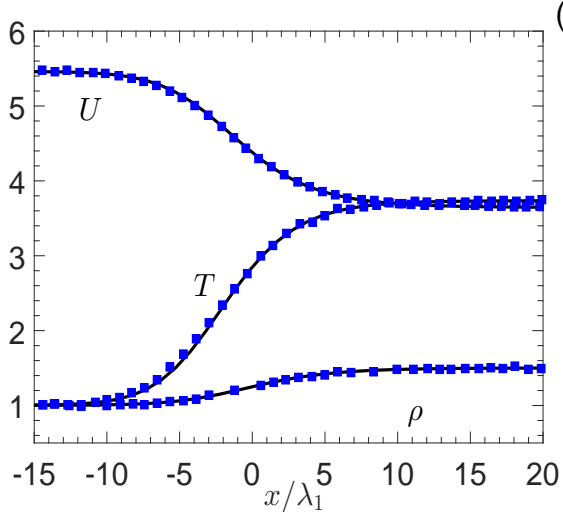

(a)

(b)

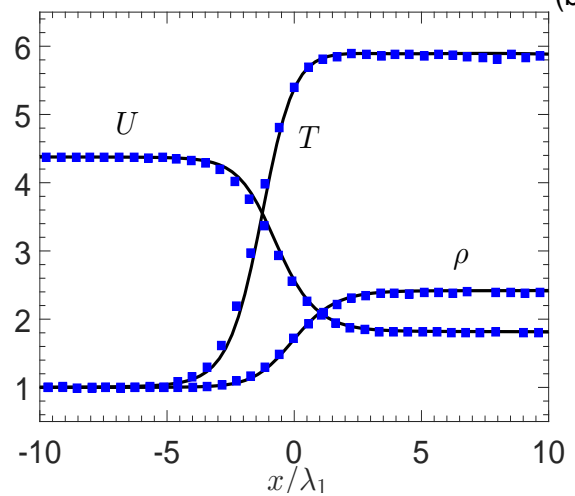

Figure 3: Comparison of $\rho, U$ and T, obtained from the Molecular Dynamics method by Frezzotti (1998) (solid square symbols) and the fast spectral method of solving the Enskog equation (solid line) at $(a) \mathrm{Ma}=3, V_{p}=1.8257$, and $E_{l}=2.9831(\eta=0.2$ ); (b) $\mathrm{Ma}=4, V_{p}=2.5674$, and $E_{l}=0.4825(\eta=0.05)$. (Note: the original MD data of the velocity and the $x$-axis in Frezzotti (1998) were presented in the units of $R T_{1}$ and $m / \sqrt{2} \pi \sigma^{2} \rho_{1}$, respectively.)

Sgarra (1993) to represent the degree of denseness in the shock upstream, is defined as the ratio of the molecular diameter $\sigma$ to the reference mean free path $\lambda_{1}=m / \sqrt{2} \pi \sigma^{2} \rho_{1} \chi\left(\rho_{1}\right)$, and is related to the upstream density $\rho_{1}$ by

$$
E_{l}=\frac{\sigma}{\lambda_{1}}=\frac{3 \sqrt{2}}{2} b \rho_{1} \chi\left(\rho_{1}\right) .
$$

Accordingly, the downstream $E_{r}$ can be obtained based on the downstream density $\rho_{2}$, which actually represents the densest part of the shock wave. The values of $E_{r}$ are shown in figure 1, where the Mach number is equal to 2 and 4 , respectively, with $E_{l}$ ranging from 0.2 to 0.8 . Here, the Mach number is defined as Ma $=U_{1} / \sqrt{\gamma R T_{1}}$, with $\gamma=5 / 3$ being the specific heat ratio for monatomic gases. We can see from figure 1 that the mean free path of molecules ranges from half to twice of the molecular diameter for the considered cases. Note that the reduced density $\eta$ is also often used to represent the degree of denseness in dense gases (Frezzotti 1998).

In our simulations, the computational domain is set to be $[-50,50]$ (in the unit of $\lambda_{1}$ ), which is uniformly meshed by 200 points. The molecular velocity space truncated at $[-15,15]$ (in the unit of $v_{m}$ ) is discretized by the mid-point rule with 200 uniform points. The macroscopic quantities $W$ are normalized as $\bar{W}=\left(W-W_{1}\right) /\left(W_{2}-W_{1}\right)$, where the subscripts ' 1 ' and ' 2 ' denote the quantities of upstream and downstream of the shock, respectively; the heat flux and shear stress are presented in the units of $\rho_{1} v_{m}^{3} / 2$ and $\rho_{1} v_{m}^{2} / 2$, respectively. Note that in what follows, the hat over $W$ has been removed for the sake of simplicity, and the $x$-axis has been shifted for all the considered quantities so that $\rho(x=0)=\left(\rho_{1}+\rho_{2}\right) / 2$.

In order to evaluate accuracy of the FSM solving the Enskog equation for normal shock wave, the normalized density, velocity, and temperature computed from the Enskog equation are compared with those of the Monte Carlo method (Frezzotti \& Sgarra 1993) as shown in figure 2, where the RDF of (2.4) is applied in this case for consistent comparison. Clearly, excellent agreements between these two methods are achieved for $\mathrm{Ma}=4$ in both the limit of dilute gas $\left(E_{l}=0\right)$ and the moderately dense gas of $E_{l}=0.2$. 
(a)
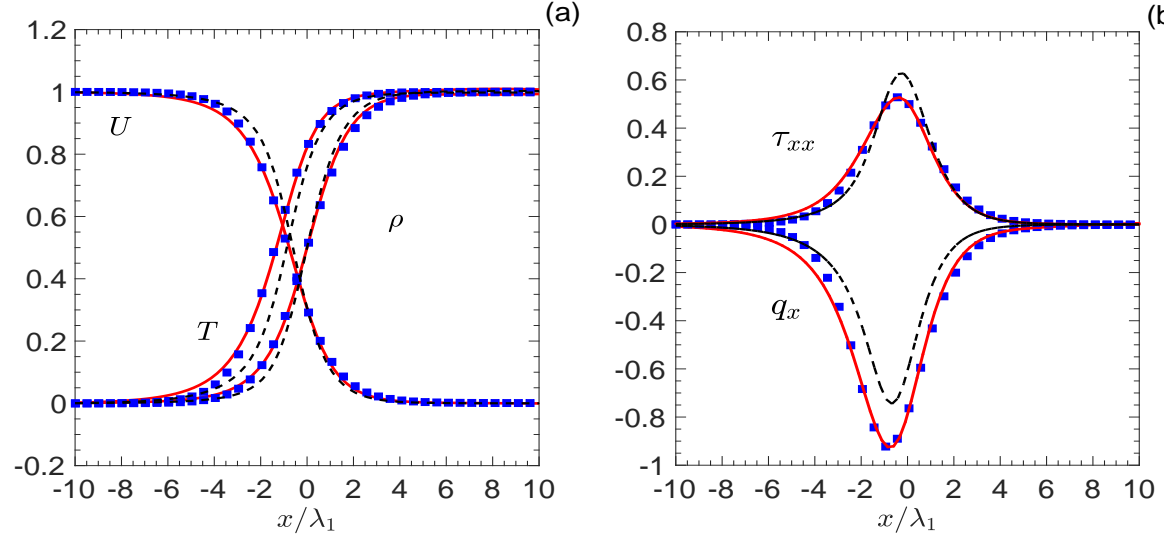

(b)

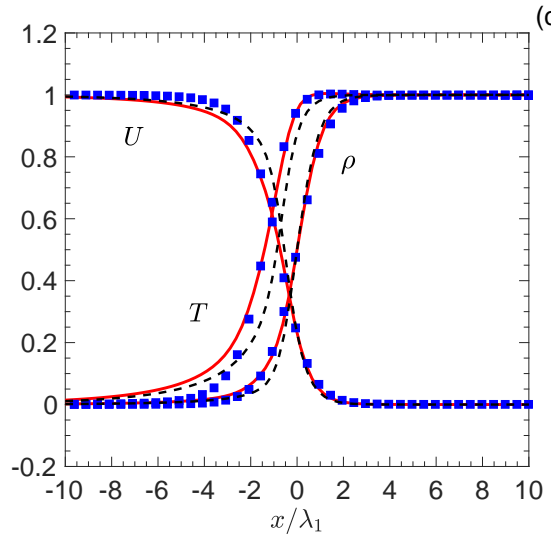

(c)

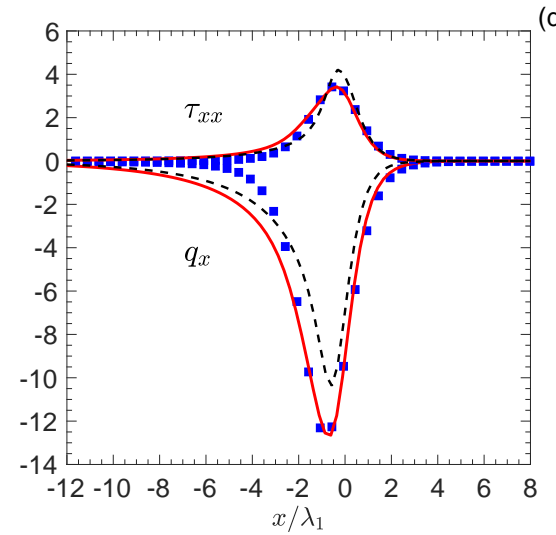

Figure 4: $(a, c)$ The normalized $\rho, U$ and $T$; and $(b, d)$ heat flux $q_{x}$ and shear stress $\tau_{x x}$. Here $E_{l}=0, \mathrm{Ma}=2$ (first row) and $\mathrm{Ma}=4$ (second row), obtained from the BGK-Enskog model (dash line), the Shakhov-Enskog model (solid line) and the Enskog equation (solid squares).

Figure 3 shows accuracy of the FSM solving the Enskog equation by comparing the computed macroscopic variables with the MD data (Frezzotti 1998). The MD simulation was conducted on a tube with two specularly reflecting end walls, one of which is at rest, whereas the other one is a piston moving at a constant speed $V_{p}$. The piston velocity of $V_{p}=1.8257$ and 2.5674 can generate shocks moving at $\mathrm{Ma}=3$ and 4 , respectively, for which the upstreaming $E_{l}=2.9831(\eta=0.2)$ and $E_{l}=0.4825(\eta=0.05)$ are simulated. It is noted from figure 3 that, even for such high degree of denseness, very good agreement between these two methods can be observed. Therefore, in what follows, solutions of the Enskog equation will be served as the benchmark to validate the proposed Shakhov-Enskog model. Note that (2.3) will be applied in the following simulations, since it strikes a balance between simplicity and accuracy (Frezzotti 1997a).

\subsection{Comparison of the Shakhov-Enskog model and BGK-Enskog model}

The normalized macroscopic variables $W$ in the limit case of dilute gases, i.e., $E_{l}=0$, for $\mathrm{Ma}=2$ and 4 computed from the Enskog equation, and the BGK-Enskog and ShakhovEnskog models are shown in figures 4(a) and 4(c); correspondingly the heat flux and translational shear stress are presented in figures $4(\mathrm{~b})$ and $4(\mathrm{~d})$. The shear stress due to 

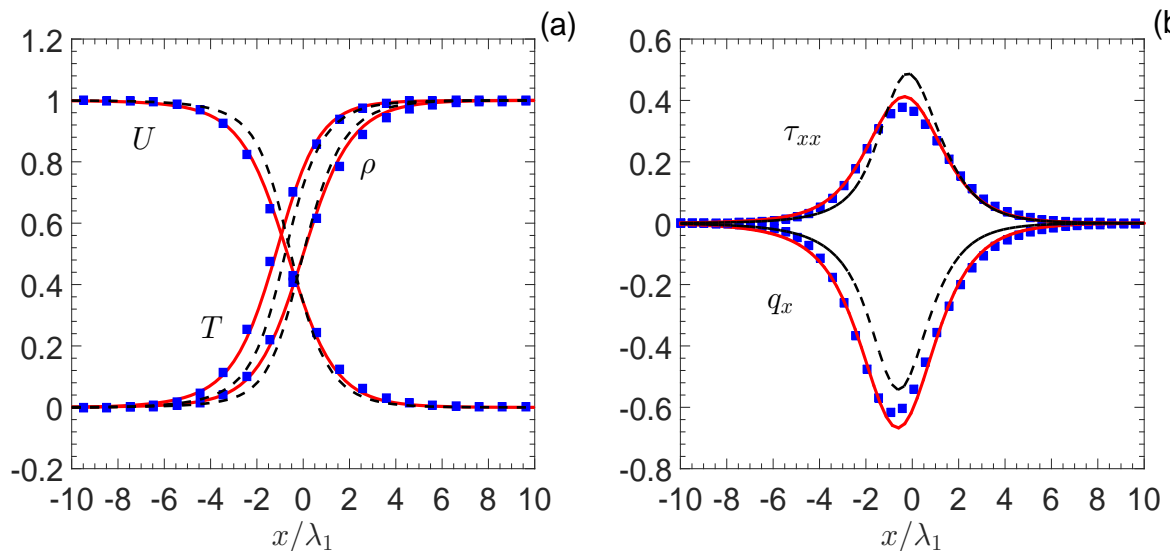

(b)

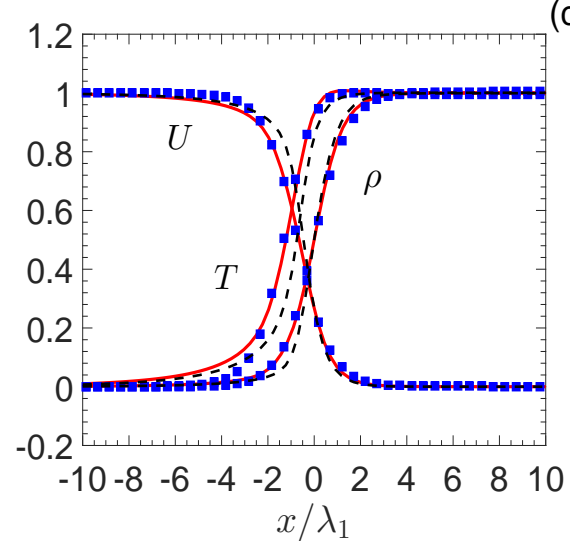

(c)

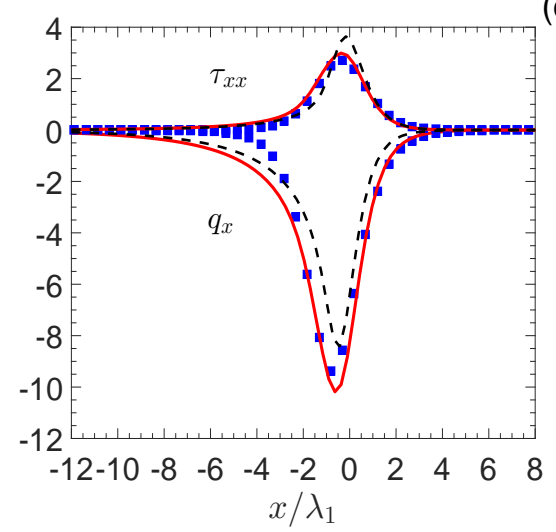

(d)

Figure 5: $(a, c)$ The normalized $\rho, U$ and $T$; and $(b, d)$ heat flux $q_{x}$ and shear stress $\tau_{x x}$. Here, $E_{l}=0.2, \mathrm{Ma}=2$ (first row) and $\mathrm{Ma}=4$ (second row), obtained from the BGK-Enskog model (dash line), the Shakhov-Enskog model (solid line) and the Enskog equation (solid squares). Note that the relation time given by (2.18) is used in both the BGK-Enskog and the Shakhov-Enskog models.

the translational motion of gas molecules $\tau_{x x}$ can be computed as

$$
\tau_{x x}=\int c_{x}^{2} f d \boldsymbol{\xi}-p_{0}
$$

When $E_{l}=0$ and $\chi$ is equal to unity, the collision operators of the Enskog equation, and the BGK-Enskog and Shakhov-Enskog models reduce to those of the Boltzmann equation, and the BGK and Shakhov models, respectively. We notice that, visible deviations appear mainly at the upstream, where degree of non-equilibrium is greater than that at the downstream due to the lower density before the shock. The BGK model shows large discrepancy from the Enskog equation not only for the temperature and heat flux that are directly relevant to the Prandtl number, but also for the density, velocity, and shear stress, while the results of the Shakhov-Enskog model agree well with those of the Enskog equation. These findings are consistent with the previous studies of shock wave structure (Li \& Zhang 2008; Xu \& Huang 2011; Li et al. 2015; Chen et al. 2015).

The similar phenomena can also be observed in figure 5 for $\mathrm{Ma}=2$ and 4 with $E_{l}=0.2$, except the early rising of temperature and heat flux in the upstream of the shock. This is 


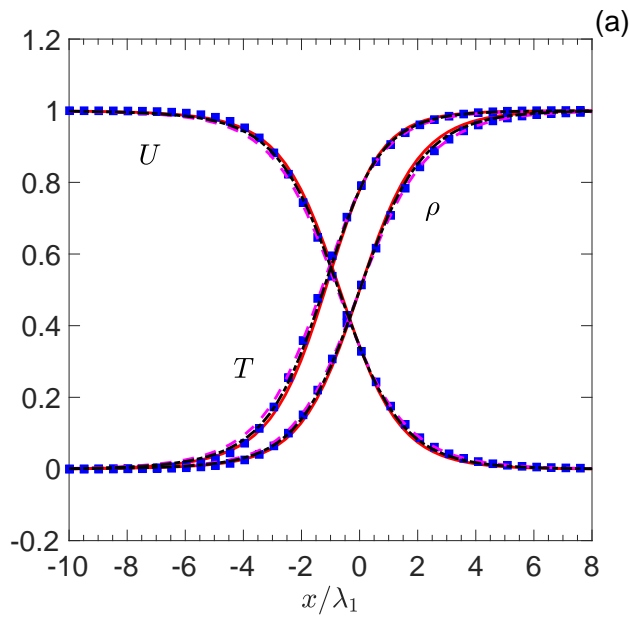

(a)

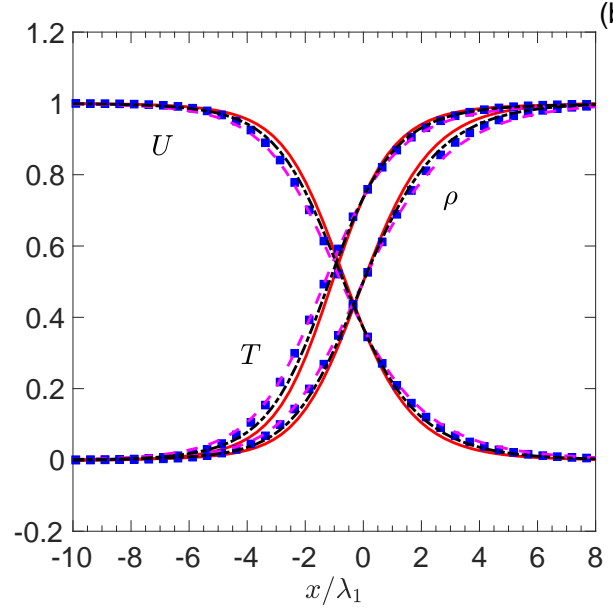

(b)

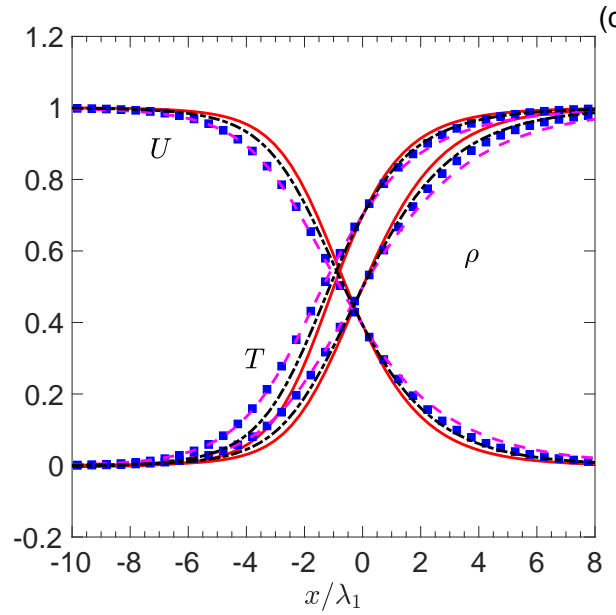

(c)

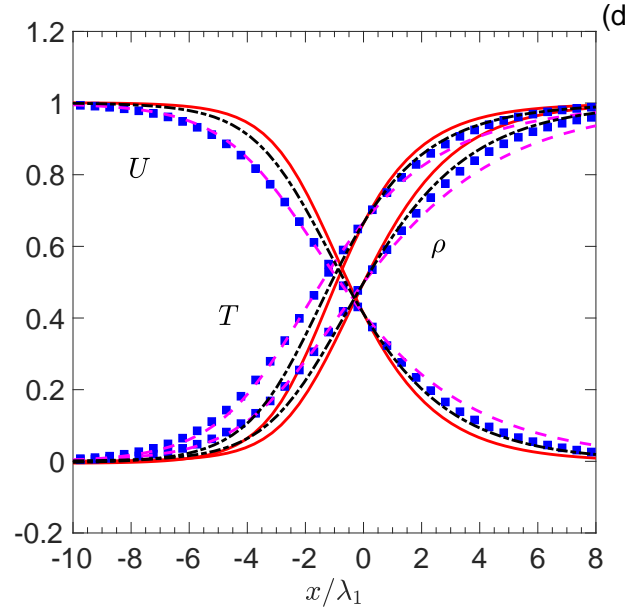

(d)

Figure 6: The profiles of normalized $\rho, U$ and $T$ at $\mathrm{Ma}=2:(a) E_{l}=0.2 ;(b) E_{l}=0.4 ;(c)$ $E_{l}=0.6$; and $(d) E_{l}=0.8$. The solid squares are the results from the Enskog equation, while the dash, solid, and dash-dot lines are the results of the Shakhov-Enskog model with the relaxation time of $(2.17),(2.18)$, and (2.19), respectively.

due to a well-acknowledged cause that the relaxation time in the model equation, which is independent of the molecular velocity, becomes less accurate for high-Mach flows. This is also the reason that the temperature field and heat flux in the case of $\mathrm{Ma}=4$ exhibit larger discrepancy than those of $\mathrm{Ma}=2$, when compared with the corresponding results of the Enskog equation, see figures 4 and 5 . For example, the maximum relative difference in the temperature for $\mathrm{Ma}=2$ and 4 is about $3 \%$ and $30 \%$, respectively.

\subsection{Evaluation of the Shakhov-Enskog model}

We now evaluate the Shakhov-Enskog model with different relaxation time of $\tilde{\tau}$ by comparing with the Enskog equation. The normalized macroscopic variables for $\mathrm{Ma}=2$ with $E_{l}$ from 0.2 to 0.8 are shown in figures $6(\mathrm{a})$-(d). It can be seen that, when $E_{l}=0.2$, the results of the Shakhov-Enskog model with the relaxation time given by $(2.17),(2.18)$ and (2.19) respectively are in good agreement with the Enskog solutions. With the increase of $E_{l}$, the deviation of the Shakhov-Enskog model from the Enskog equation 

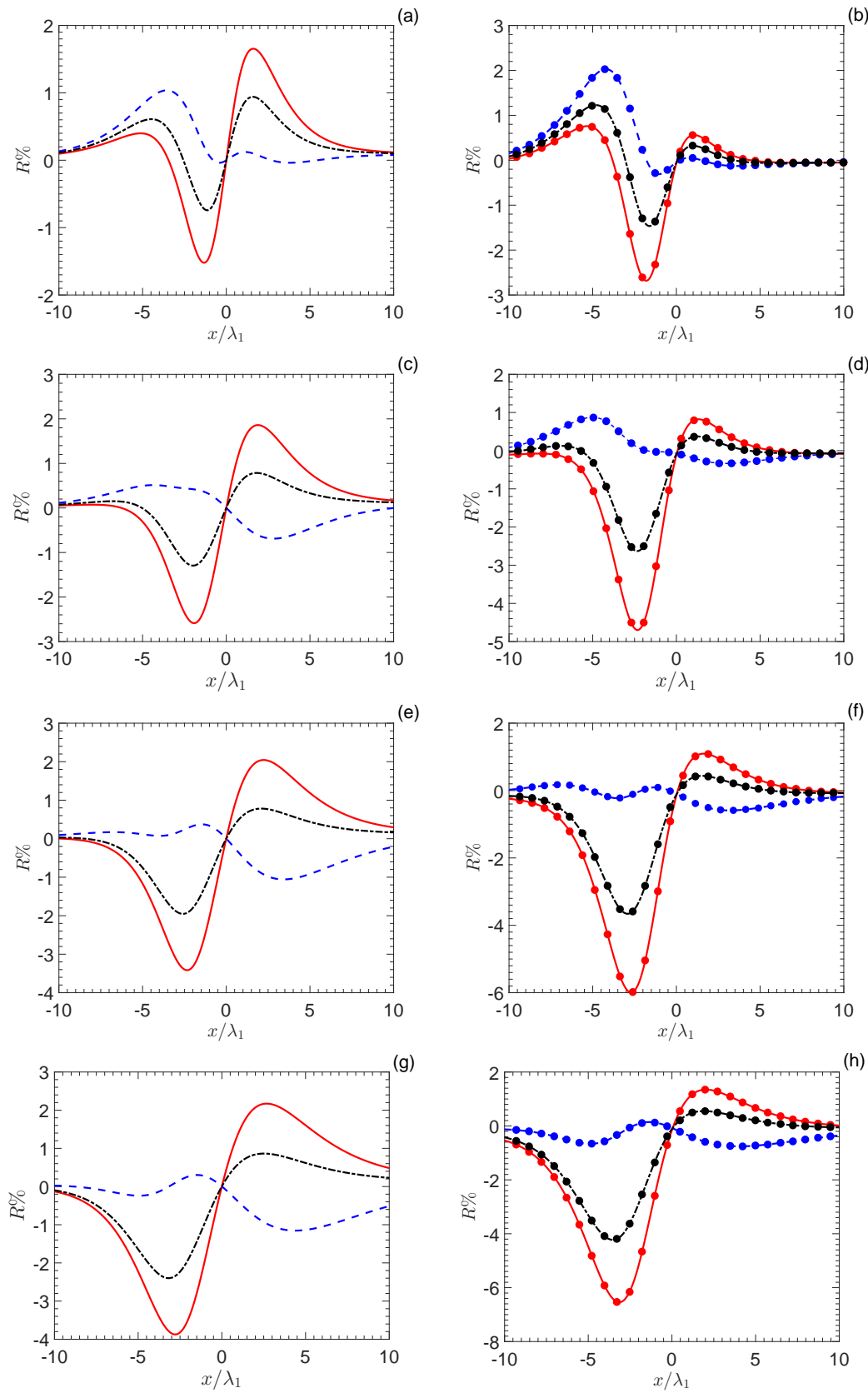

Figure 7: The relative difference $R(\%)\left(R=100\left[W_{\text {Shakhov-Enskog }} / W_{\text {Enskog }}-1\right]\right)$ for the density (left row) and the temperature (right row) at $\mathrm{Ma}=2:(a, b) E_{l}=0.2 ;(c, d)$ $E_{l}=0.4 ;(e, f) E_{l}=0.6$; and $(g, h) E_{l}=0.8$. $W_{\text {Shakhov-Enskog }}$ and $W_{\text {Enskog }}$ represent the results from the Shakhov-Enskog model and the Enskog equation, respectively. In the left row of the figure, the dash, solid, and dash-dot lines are the relative density difference for the Shakhov-Enskog model using (2.17), (2.18), and (2.19), respectively. Accordingly, in the right row of the figure, these lines with the solid circles are the relative temperature difference. 
(a)
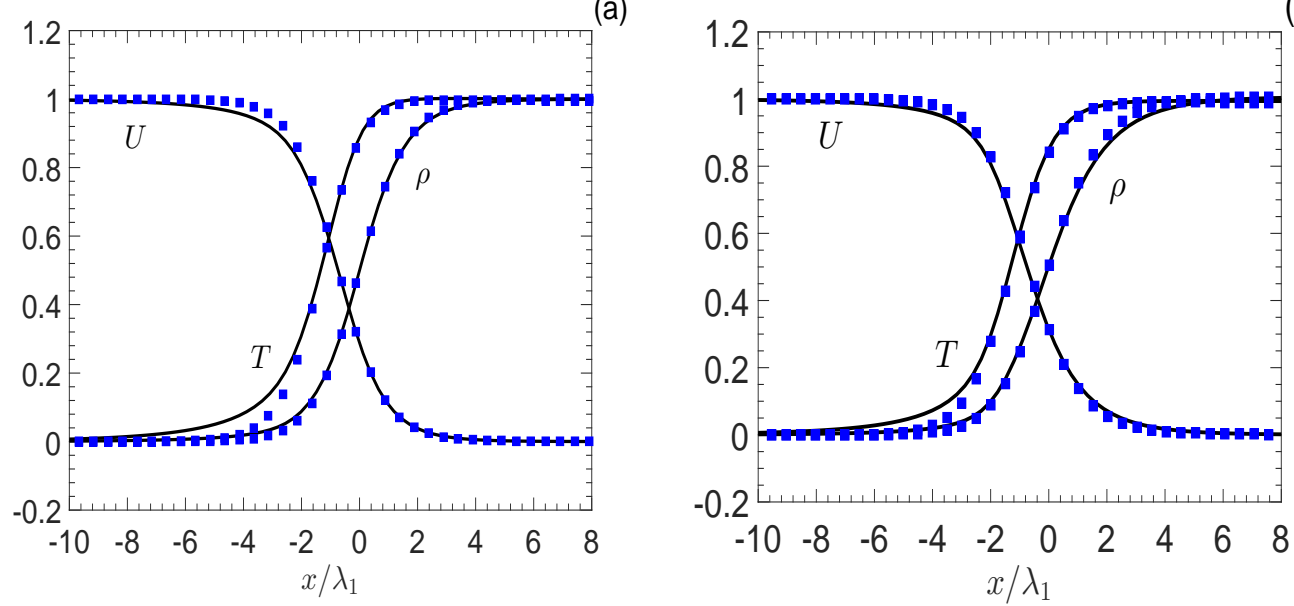

(b)

(c)
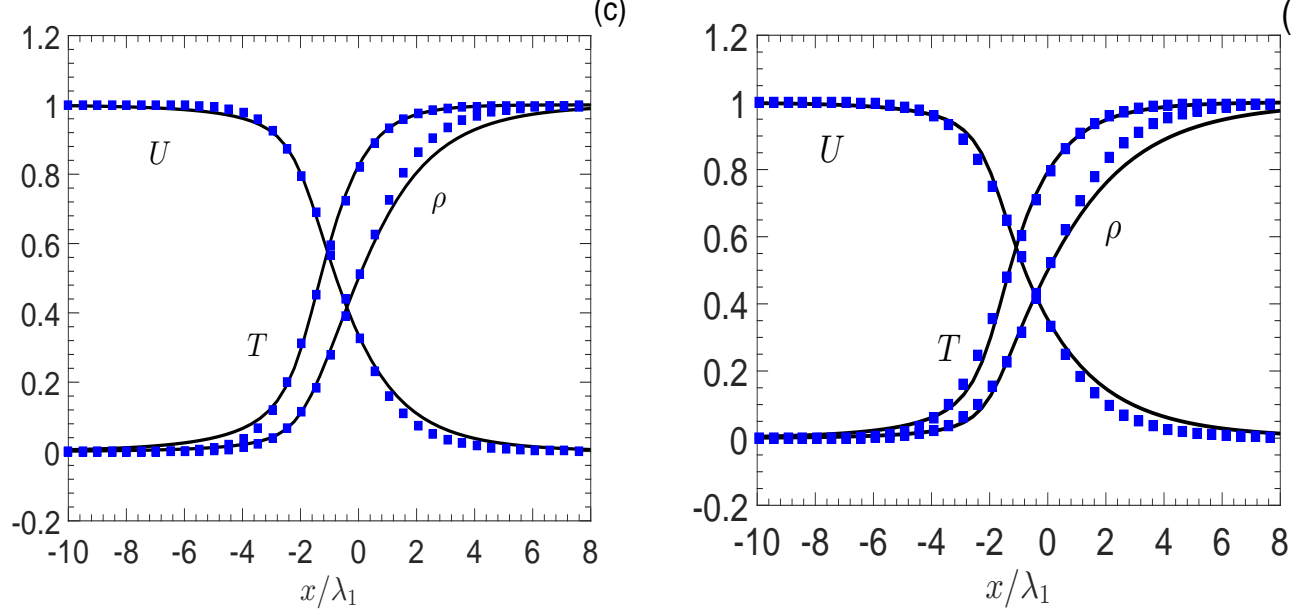

(d)

Figure 8: The profiles of normalized $\rho, U$ and $T$ at $\mathrm{Ma}=3$, with $(a) E_{l}=0.2 ;(b) E_{l}=0.4$; (c) $E_{l}=0.6$; and $(d) E_{l}=0.8$. The solid squares are the results from the Enskog equation, while the solid lines are the results of the Shakhov-Enskog model with the relaxation time of (2.17).

becomes more pronounced. We notice that for the case of $E_{l}=0.4$, the profiles calculated from the proposed model with the relaxation time of (2.18) show more visible difference from those of the Enskog equation, while the results computed from the model with (2.17) and (2.19) still show a good agreement with the reference solutions. However, when $E_{l} \geqslant 0.6$, both $(2.18)$ and $(2.19)$ cannot reproduce acceptable shock structure profiles, particularly at the upstream where the Shakhov-Enskog model clearly underestimates the macroscopic variables. When using (2.17), as expected, the kinetic model overall shows a better agreement with the Enskog equation for all the values of $E_{l}$; in particular, the early rise of temperature in the upstream seems to be accurately captured by the Shakhov-Enskog model.

The performance of the Shakhov-Enskog model with different formulae of the relaxation time can be quantitatively explained by the relative difference in density and temperature, which is shown in the left and right rows of figure 7 , respectively. Note that the relative difference in velocity lies between those of the density and temperature, 


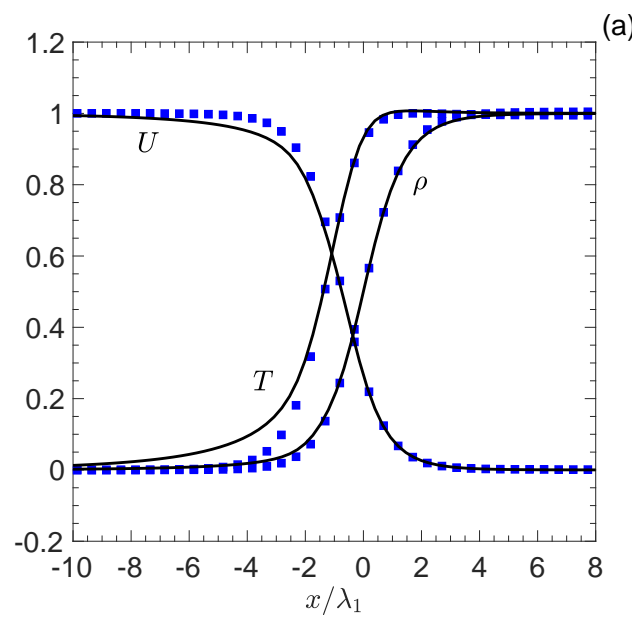

(c)

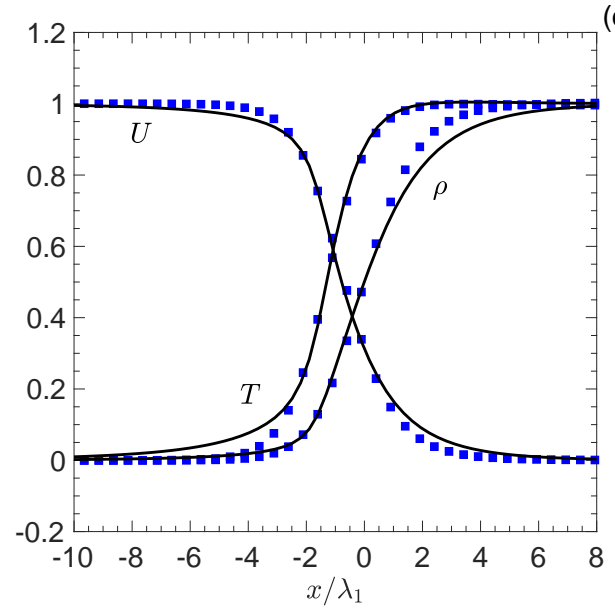

(a)

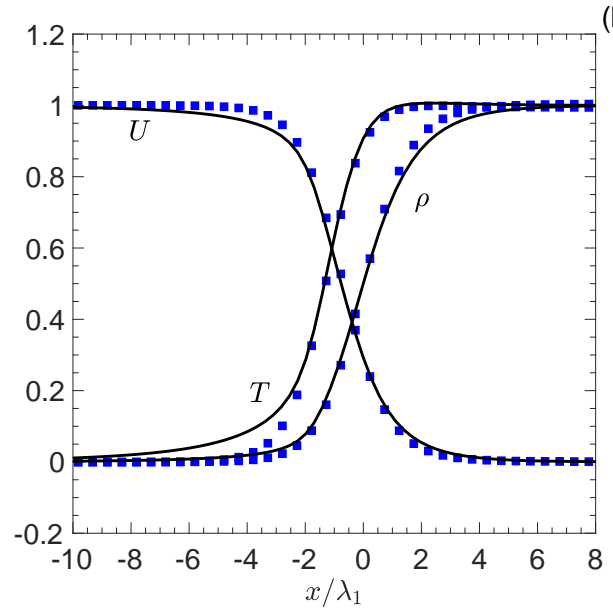

(d)

Figure 9: The profiles of normalized $\rho, U$ and $T$ at Ma=4, with $(a) E_{l}=0.2 ;(b) E_{l}=0.4$; (c) $E_{l}=0.6$; and $(d) E_{l}=0.8$. The solid squares are the results from the Enskog equation, while the solid lines are the results of the Shakhov-Enskog model with the relaxation time of $(2.17)$.

which is not presented for clarity. It is clearly found that, in the most region of the shock structure, the maximum relative difference in the macroscopic variables computed from the present model with (2.17) is less than $2 \%$, which is the smallest amongst these three formulae. When using the relaxation time of (2.18), the obtained temperature could differ from the Enskog solution by $6 \%$ at $E_{l}=0.6$; meanwhile, the model with (2.19) can also overestimate the temperature by $4 \%$ at $E_{l}=0.8$. The above results show that the proposed Shakhov-Enskog model with the relaxation time of (2.17), which adopts the viscosity coefficient accounting for both kinetic and potential contributions, expectedly provides the best prediction of the shock structure. Therefore, unless otherwise stated, the relaxation time of (2.17) will be applied in the Shakhov-Enskog model in the following simulations.

The results obtained from the Shakhov-Enskog model with (2.17) and the Enskog equation are further presented in figures 8 and 9 for $\mathrm{Ma}=3$ and $\mathrm{Ma}=4$, respectively. With the increase of $E_{l}$, despite the growing underestimate of the density near the shock 
(a)
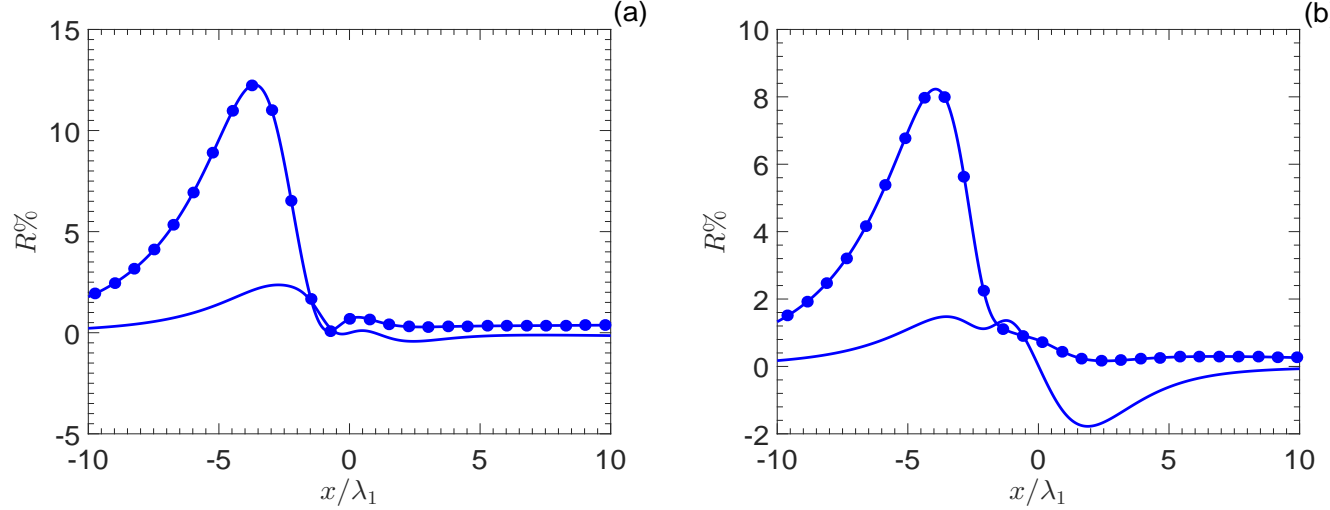

(b)
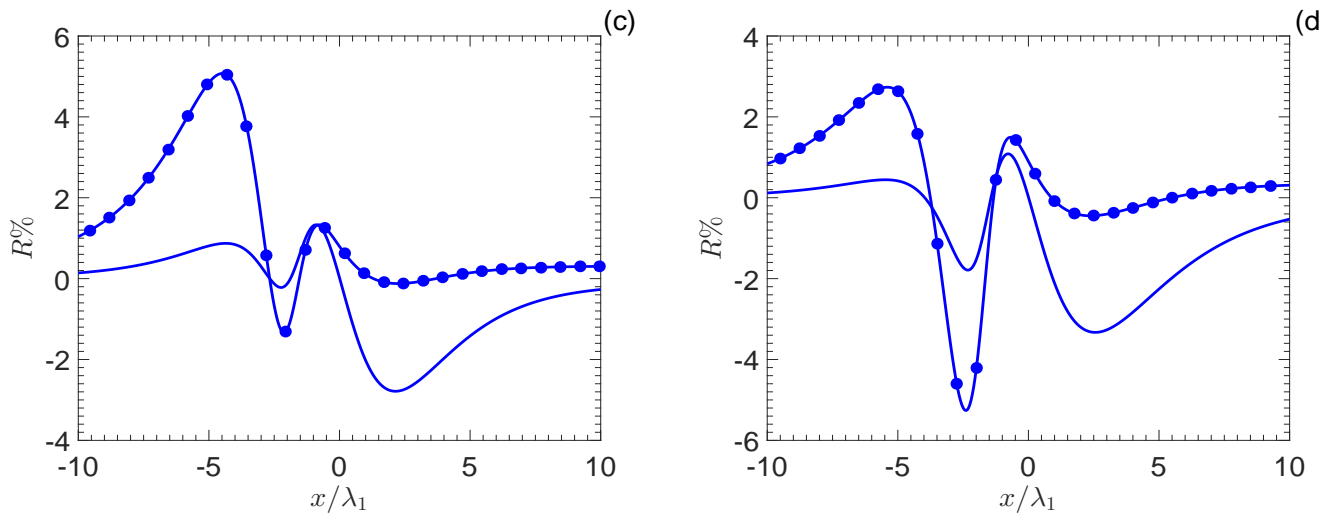

Figure 10: The relative difference in the macroscopic quantities $R(\%)$ for $\mathrm{Ma}=3$, obtained from the Shakhov-Enskog model using the relaxation time of $(2.17):(a) E_{l}=0.2 ;(b) E_{l}=$ $0.4 ;(c) E_{l}=0.6$; and $(d) E_{l}=0.8$. The relative difference in the density and temperature are represented by the solid line and the solid line with solid circles, respectively.

front $\left(x / \lambda_{1}>2\right)$, overall good agreements between both sets of results are observed. The same as the case of $\mathrm{Ma}=2$, for such high Mach number flows, conspicuous overestimate of the temperature by the present model arises before the shock front as well; this is due to the inherent drawback of the Shakhov model. Meanwhile, it is interesting to see that, the overestimate of the temperature becomes less significant when the Mach number increases, which may be attributed to the reducing non-equilibrium effect due to the increase of $E_{l}$ at the shock upstream.

The discrepancy between the proposed model and the Enskog equation is further examined by the relative differences in the shock profiles, which are shown in figures 10 and 11 for the cases of $\mathrm{Ma}=3$ and 4, respectively. Note that the relative difference in the velocity has the same value with that of the density but with an opposite sign, which is not presented. We can see that, the Shakhov-Enskog model can reproduce the density and velocity profiles accurately, with an overall relative difference being smaller than $4 \%$ and $10 \%$ for $\mathrm{Ma}=3$ and 4 , respectively, whereas there exhibits large deviation in the temperature profile from the Enskog solution by a maximum relative difference up to $15 \%$ and $30 \%$, respectively, at $E_{l}=0.2$. Meanwhile, as clearly shown, the maximum difference of temperature is smaller at a larger $E_{l}$. This deficiency of the proposed model is inherited from the Shakhov collision operator itself, instead of the Enskog equation. However, the relative difference in the density and velocity profiles is less than $5 \%$, as shown in figures 7 , 

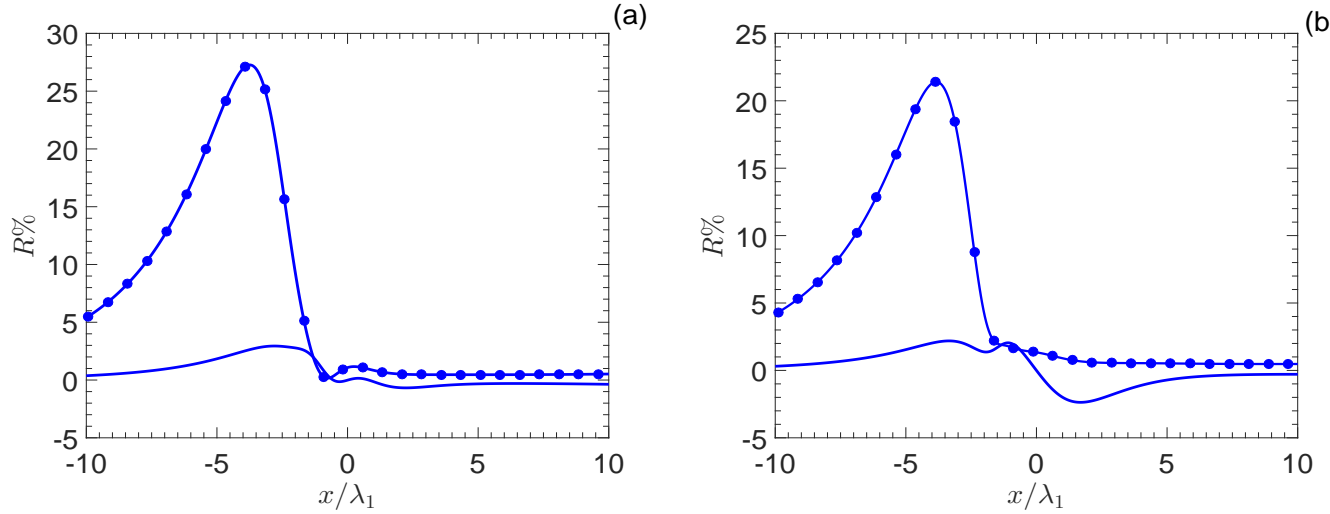

(b)

(c)
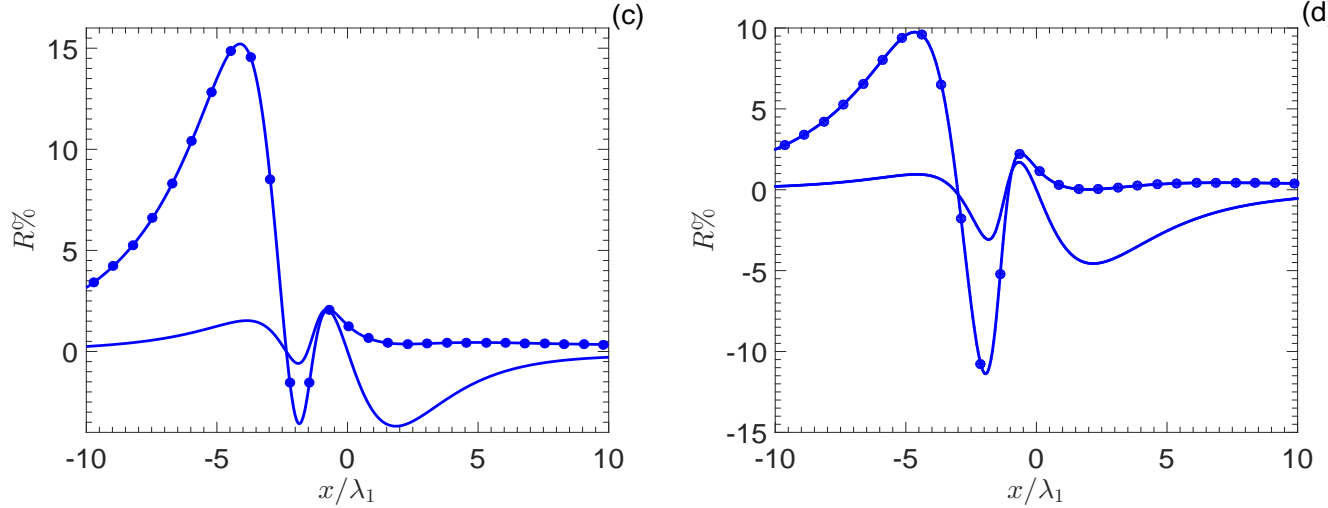

Figure 11: The relative difference in the macroscopic quantities $(R \%)$ for $\mathrm{Ma}=4$ obtained from the Shakhov-Enskog model using the relaxation time of $(2.17)$ : $(a) E_{l}=0.2 ;(b) E_{l}=$ $0.4 ;(c) E_{l}=0.6$; and $(d) E_{l}=0.8$. The relative difference in the density and temperature are represented by the solid line and the solid line with solid circles, respectively.

10, and 11, suggesting that the Shakhov-Enskog model can capture rarefaction effects reasonably well in dense gases. For very dense gases where the molecules are nearly in constant contact, both the Enskog equation and its model become inaccurate, which is reflected in the results for large $E_{l}$.

The differential performance of the Shakhov-Enskog model when using different types of relaxation time can be essentially explained from the microscopic VDF. The parallel VDFs as a function of $\xi_{x}$ at different locations of the shock are shown in figure 12, where the Shakhov-Enskog model with the relaxation time of (2.17) and (2.18) for $\mathrm{Ma}=4$ and $E_{l}=0.4$ are examined as an example. The results are similar for the other parameters. It is noticed that, the VDFs computed from the Shakhov-Enskog model with (2.17) are better than the model with (2.18), when compared with the Enskog equation; particularly in the region of $\xi_{x} \in\left(-v_{m}, 2 v_{m}\right)$; see figures $12(\mathrm{a})$ and $12(\mathrm{~b})$. In addition, as we see from figure $12(\mathrm{~d})$, at $x / \lambda_{1}=10$ where it is far from the shock center, the proposed model can accurately reproduce VDF close to equilibrium. Note that one of the potential drawbacks inherited from the Shakhov collision operator, i.e., the negative value of VDF, does not appear in the above simulations. 

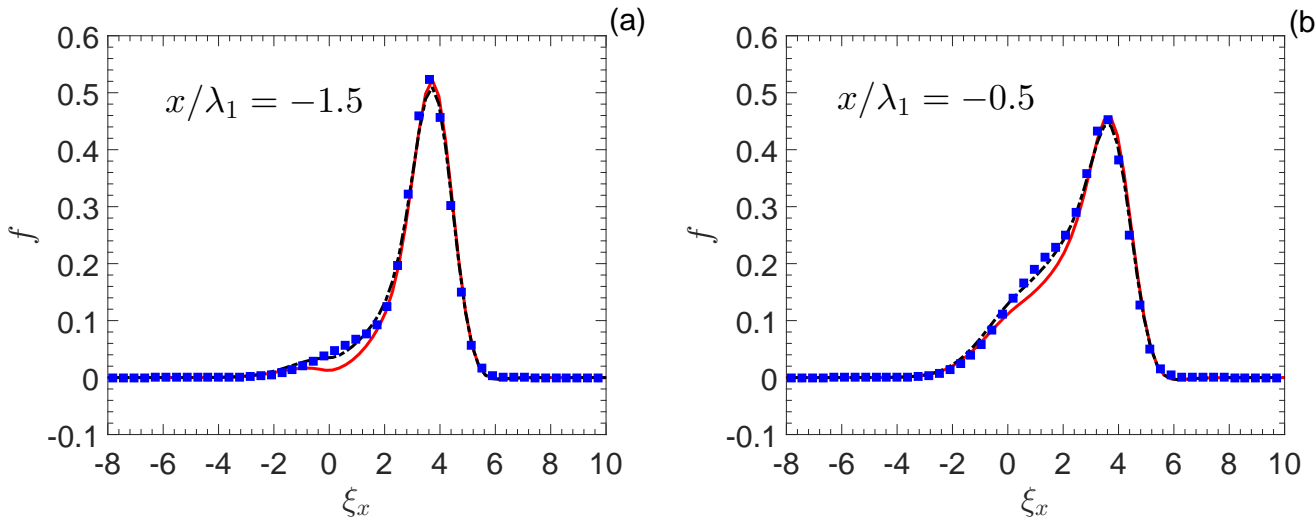

(b)

(c)
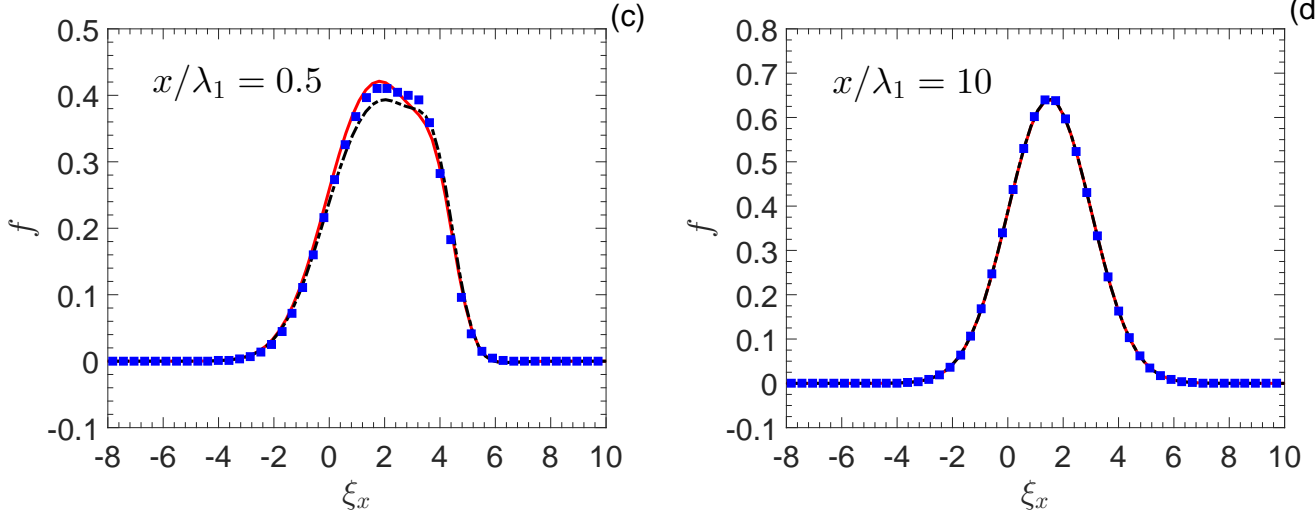

(d)

Figure 12: The marginal reduced velocity distribution function $f$ as a function of the molecular velocity $\xi_{x}$ at $x / \lambda_{1}=-1.5,-0.5,0.5,10$. The solid squares are the results from the Enskog equation, while the dash-dot and solid lines are the results of the ShakhovEnskog model with the relaxation time of (2.17) and (2.18), respectively. The molecular velocity $\xi_{x}$ is in the unit of most probable velocity $v_{m}$.

\section{Concluding remarks}

In this work, a kinetic model has been proposed to simplify the Enskog equation for non-equilibrium flow of dense gases using the kinetic Shakhov model which can recover the correct Prandtl number of monatomic dilute gases. The propagation of normal shock wave in a dense gas is simulated to validate the proposed Shakhov-Enskog model against the FSM solution of the Enskog equation. In comparison with the BGK-Enskog model, the Shakhov-Enskog model with a correct Prandtl number agrees better with the Enskog equation, not only for the temperature and heat flux that are directly relevant to the Prandtl number, but also for the density, velocity, and shear stress.

The relaxation time of (2.17) accounts for both the kinetic and collisional contributions to the momentum and energy transfer, so the Shakhov-Enskog model can predict the shock profiles well. Surprisingly, with the use of (2.17), the early rising of temperature as observed in the dilute gases is also much improved.

In conclusion, the proposed Shakhov-Enskog model is capable of describing the momentum and energy transfer inside shock, when the maximum local mean free path of gas molecules is greater than the size of gas molecules. More importantly, for all the simulations in this study, the computational cost for the DVM solution of the Shakhov- 
Enskog model is at least 2-order less than the FSM solution of the Enskog equation. The current work does not consider the gas-surface interactions, which are an important but difficult research challenge for surface-bounded non-equilibrium flow of dense gases. When the attractive force between molecules is properly considered, the Shakhov-Enskog model may also provide a new way of modeling non-equilibrium behavior of multiphase flows.

\section{ACKNOWLEDGMENT}

Financial support from the UK Engineering and Physical Sciences Research Council (EPSRC) under Grants EP/M021475/1, EP/R041938/1, and EP/R029598/1 is gratefully acknowledged. This work is also supported by the College of Petroleum Engineering and Geosciences, King Fahd University of Petroleum and Minerals, Saudi Arabia.

\section{Appendix A. Reduction of the Shakhov-Enskog model for one-dimensional case}

Since only one-dimensional shock wave is considered in this study, two reduced velocity distribution functions are introduced to cast the three-dimensional molecular velocity space $\left(\xi_{x}, \xi_{y}, \xi_{z}\right)$ into one dimension as

$$
\begin{aligned}
g & =\int f(\boldsymbol{x}, \boldsymbol{\xi}, t) \mathrm{d} \xi_{y} \mathrm{~d} \xi_{z}, \\
h & =\int\left(\xi_{y}^{2}+\xi_{z}^{2}\right) f(\boldsymbol{x}, \boldsymbol{\xi}, t) \mathrm{d} \xi_{y} \mathrm{~d} \xi_{z} .
\end{aligned}
$$

Once $g$ and $h$ are obtained, the macroscopic variables can be computed as

$$
\rho=\int g \mathrm{~d} \xi_{x}, \quad \rho U=\int \xi_{x} g \mathrm{~d} \xi_{x}, \quad T=\frac{1}{3 \rho R} \int\left(c_{x}^{2} g+h\right) \mathrm{d} \xi_{x},
$$

and the high-order moments of interest, i.e. the shear stress $\tau_{x x}$ and heat flux $q_{x}$, which are given by

$$
\tau_{x x}=\int c_{x}^{2} g-\rho R T, \quad q_{x}=\frac{1}{2} \int c_{x}\left(c_{x}^{2} g+h\right) \mathrm{d} \xi_{x} .
$$

The governing equations for the two reduced VDFs can be deduced from (2.13) as

$$
\begin{aligned}
& \frac{\partial g}{\partial t}+\xi_{x} \frac{\partial g}{\partial x}=-\frac{1}{\tilde{\tau}}\left[g-g^{S}\right]+J_{a, g}^{1}+J_{b, g}^{1}, \\
& \frac{\partial h}{\partial t}+\xi_{x} \frac{\partial h}{\partial x}=-\frac{1}{\tilde{\tau}}\left[h-h^{S}\right]+J_{a, h}^{1}+J_{b, h}^{1}
\end{aligned}
$$

where the reduced reference VDFs are $g^{S}=g^{e q}+g_{\operatorname{Pr}}$ and $h^{S}=h^{e q}+h_{\mathrm{Pr}}$, with

$$
\begin{aligned}
g^{e q} & =\frac{\rho}{(2 \pi R T)^{1 / 2}} \exp \left[-\frac{c_{x}^{2}}{2 R T}\right], \\
h^{e q} & =2 R T g^{e q}, \\
g_{\operatorname{Pr}} & =(1-\operatorname{Pr}) \frac{c_{x} q_{x}}{5 p_{0} R T}\left[\frac{c_{x}^{2}}{R T}-3\right] g^{e q}, \\
h_{\operatorname{Pr}} & =(1-\operatorname{Pr}) \frac{c_{x} q_{x}}{5 p_{0} R T}\left[\frac{c_{x}^{2}}{R T}-1\right] h^{e q},
\end{aligned}
$$


and

$$
\begin{aligned}
& J_{a, g}^{1}=-g^{e q} b \rho c_{x} \partial_{x} \chi \\
& J_{b, g}^{1}=-g^{e q} b \rho \chi\left\{2 c_{x} \partial_{x} \ln \rho+\frac{3}{5}\left[\frac{c_{x}^{2}}{R T}-1\right] \partial_{x} u_{x}+\frac{3}{10}\left[\frac{c_{x}^{3}}{R T}+\frac{1}{3} c_{x}\right] \partial_{x} \ln T\right\} \\
& J_{a, h}^{1}=-h^{e q} b \rho c_{x} \partial_{x} \chi \\
& J_{b, h}^{1}=-h^{e q} b \rho \chi\left\{2 c_{x} \partial_{x} \ln \rho+\frac{3}{5}\left[\frac{c_{x}^{2}}{R T}-\frac{1}{3}\right] \partial_{x} u_{x}+\frac{3}{10}\left[\frac{c_{x}^{3}}{R T}+\frac{7}{3} c_{x}\right] \partial_{x} \ln T\right\},
\end{aligned}
$$

where $\partial_{x}$ denotes the derivative with respect to the $x$-axis. It is clear that the governing equation for $g$ and $h$ in (A 4 ) can be expressed in the following compact form:

$$
\frac{\partial \phi}{\partial t}+\xi_{x} \frac{\partial \phi}{\partial x}=-\frac{1}{\tilde{\tau}}\left[\phi-\phi^{S}\right]+\Omega_{E, \phi},
$$

where the symbol $\phi$ is used to represent $g$ or $h$, and $\Omega_{E, \phi}=J_{a, \phi}^{1}+J_{b, \phi}^{1}$. Finally, (A 7) is readily solved by the iterative implicit DVM described by (3.2).

\section{REFERENCES}

Alder, Berni Julian \& Wainwright, Thomas Everett 1960 Studies in molecular dynamics. ii. behavior of a small number of elastic spheres. The Journal of Chemical Physics 33 (5), $1439-1451$.

Alexander, Francis J, Garcia, Alejandro L \& Alder, Berni J 1995 A consistent Boltzmann algorithm. Physical Review Letters 74 (26), 5212.

Bhatnagar, Prabhu Lal, Gross, Eugene P \& Krook, Max 1954 A model for collision processes in gases. I. small amplitude processes in charged and neutral one-component systems. Physical Review 94 (3), 511.

Brenner, Michael P, Hilgenfeldt, Sascha \& Lohse, Detlef 2002 Single-bubble sonoluminescence. Reviews of Modern Physics $\mathbf{7 4}$ (2), 425.

Carnahan, Norman F \& Starling, Kenneth E 1969 Equation of state for nonattracting rigid spheres. The Journal of Chemical Physics 51 (2), 635-636.

Chapman, Sydney \& Cowling, Thomas George 1970 The mathematical theory of nonuniform gases: an account of the kinetic theory of viscosity, thermal conduction and diffusion in gases. Cambridge university press.

Chen, Songze, Xu, Kun \& CaI, Qingdong 2015 A comparison and unification of ellipsoidal statistical and Shakhov BGK models. Advances in Applied Mathematics and Mechanics 7 (2), 245-266.

Dahms, Rainer N, Manin, Julien, Pickett, Lyle M \& Oefelein, Joseph C 2013 Understanding high-pressure gas-liquid interface phenomena in diesel engines. Proceedings of the Combustion Institute 34 (1), 1667-1675.

Dahms, Rainer N \& Oefelein, Joseph C 2015 Non-equilibrium gas-liquid interface dynamics in high-pressure liquid injection systems. Proceedings of the Combustion Institute 35 (2), $1587-1594$.

Enskog, David 1922 Kinetische Theorie der Wärmeleitung: Reibung und Selbst-diffusion in Gewissen verdichteten gasen und flüssigkeiten. Almqvist \& Wiksells boktryckeri-a.-b.

Ferziger, Joel H \& Kaper, Hans G 1972 Mathematical theory of transport processes in gases. North-Holland.

Frezzotti, Aldo 1997a Molecular dynamics and Enskog theory calculation of one dimensional problems in the dynamics of dense gases. Physica A: Statistical Mechanics and its Applications 240 (1-2), 202-211.

Frezzotti, Aldo 1997b A particle scheme for the numerical solution of the Enskog equation. Physics of Fluids 9 (5), 1329-1335.

FrezzotTi, Aldo 1998 Molecular dynamics and enskog theory calculation of shock profiles in a dense hard sphere gas. Computers $\&$ Mathematics with Applications 35 (1-2), 103-112.

Frezzotti, Aldo \& Sgarra, Carlo 1993 Numerical analysis of a shock-wave solution of 
the Enskog equation obtained via a Monte Carlo method. Journal of Statistical Physics 73 (1-2), 193-207.

Guo, Zhaoli \& Shu, Chang 2013 Lattice Boltzmann method and its applications in engineering. World Scientific.

Guo, Zhaoli, Zhao, TS \& Shi, Yong 2005 Simple kinetic model for fluid flows in the nanometer scale. Physical Review E 71 (3), 035301.

Guo, Zhaoli, Zhao, TS \& Shi, Yong 2006 Generalized hydrodynamic model for fluid flows: From nanoscale to macroscale. Physics of Fluids 18 (6), 067107.

de Haro, M López \& Garzó, V 1995 Shock waves in a dense gas. Physical Review E 52 (5), 5688.

He, XiaOyi \& Doolen, Gary D 2002 Thermodynamic foundations of kinetic theory and lattice Boltzmann models for multiphase flows. Journal of Statistical Physics 107 (1-2), 309-328.

Kremer, GiLberto M 2010 An introduction to the Boltzmann equation and transport processes in gases. Springer Science \& Business Media.

Li, Zhi-Hui, Peng, Ao-Ping, Zhang, Han-Xin \& Yang, Jaw-Yen 2015 Rarefied gas flow simulations using high-order gas-kinetic unified algorithms for Boltzmann model equations. Progress in Aerospace Sciences 74, 81-113.

Li, Zhi-Hui \& Zhang, Han-Xin 2008 Gas-kinetic description of shock wave structures by solving Boltzmann model equation. International Journal of Computational Fluid Dynamics 22 (9), 623-638.

LuO, LI-SHI 1998 Unified theory of lattice Boltzmann models for nonideal gases. Physical Review Letters 81 (8), 1618.

Luo, LI-ShI 2000 Theory of the lattice Boltzmann method: Lattice Boltzmann models for nonideal gases. Physical Review E 62 (4), 4982.

Montanero, JM, de Haro, M López, Garzo, V \& Santos, A 1998 Strong shock waves in a dense gas: Burnett theory versus Monte Carlo simulation. Physical Review E 58 (6), 7319 .

Montanero, JM, de Haro, M Lopez, Santos, A \& Garzo, V 1999 Simple and accurate theory for strong shock waves in a dense hard-sphere fluid. Physical Review E 60 (6), 7592 .

Montanero, José María \& Santos, Andrés 1996 Monte Carlo simulation method for the Enskog equation. Physical Review E 54 (1), 438.

Petersen, Eric L \& Hanson, Ronald K 2001 Nonideal effects behind reflected shock waves in a high-pressure shock tube. Shock Waves 10 (6), 405-420.

SADR, M \& GORJI, MH 2017 A continuous stochastic model for non-equilibrium dense gases. Physics of Fluids 29 (12), 122007.

Sadr, Mohsen \& GorJi, M Hossein 2019 Treatment of long-range interactions arising in the Enskog-Vlasov description of dense fluids. Journal of Computational Physics 378, 129-142.

Sander, Regina, Pan, Zhejun \& Connell, Luke D 2017 Laboratory measurement of low permeability unconventional gas reservoir rocks: A review of experimental methods. Journal of Natural Gas Science and Engineering 37, 248-279.

ShakHov, EM 1968 Generalization of the Krook kinetic relaxation equation. Fluid Dynamics 3 (5), 95-96.

Titarev, VA 2018 Application of model kinetic equations to hypersonic rarefied gas flows. Computers \& Fluids 169, 62-70.

Venugopal, Vishnu, Praturi, Divya Sri \& Girimaji, Sharath S 2019 Non-equilibrium thermal transport and entropy analyses in rarefied cavity flows. Journal of Fluid Mechanics 864, 995-1025.

Wu, Lei, Liu, Hainu, Reese, Jason M \& Zhang, Yonghao 2016 Non-equilibrium dynamics of dense gas under tight confinement. Journal of Fluid Mechanics 794, 252-266.

Wu, Lei, White, Craig, Scanlon, Thomas J, Reese, Jason M \& Zhang, Yonghao 2013 Deterministic numerical solutions of the Boltzmann equation using the fast spectral method. Journal of Computational Physics 250, 27-52.

Wu, Lei, Zhang, Yonghao \& Reese, Jason M 2015 Fast spectral solution of the generalized Enskog equation for dense gases. Journal of Computational Physics 303, 66-79. 
Xu, Kun \& Huang, Juan-Chen 2011 An improved unified gas-kinetic scheme and the study of shock structures. IMA Journal of Applied Mathematics 76 (5), 698-711.

YANG, JY \& HUANG, JC 1995 Rarefied flow computations using nonlinear model Boltzmann equations. Journal of Computational Physics 120 (2), 323-339.

Zhu, Yajun, Zhong, Chengwen \& Xu, Kun 2019 An implicit unified gas-kinetic scheme for unsteady flow in all knudsen regimes. Journal of Computational Physics 386, 190-217. 